Voix et Images

\title{
Mythocritique, mythanalyse et littérature québécoise
}

\section{Jean Levasseur}

Volume 19, numéro 3 (57), printemps 1994

Science et fiction au Québec : L'émergence d'un savoir

URI : https://id.erudit.org/iderudit/201123ar

DOI : https://doi.org/10.7202/201123ar

Aller au sommaire du numéro

Éditeur(s)

Université du Québec à Montréal

ISSN

0318-9201 (imprimé)

1705-933X (numérique)

Découvrir la revue

Citer cet article

Levasseur, J. (1994). Mythocritique, mythanalyse et littérature québécoise. Voix et Images, 19(3), 636-640. https://doi.org/10.7202/201123ar d'utilisation que vous pouvez consulter en ligne.

https://apropos.erudit.org/fr/usagers/politique-dutilisation/ 


\title{
Mythocritique, mythanalyse et littérature québécoise
}

\author{
Jean Levasseur, Université Bishop's
}

Mythocritique et mythanalyse ne font encore que peu d'adeptes au Québec; à part quelques études de Jacques Blais (Saint-Denys Garneau), Maurice Émond et Denis Bouchard (Anne Hébert), Victor Taboul (mythe du Juif), Jack Warwick (les grands thèmes de la littérature québécoise) et quelques autres ${ }^{1}$, peu d'auteurs s'étaient aventurés jusqu'à maintenant dans l'analyse de textes littéraires via une approche où mythe et culture se conjuguent pour tenter d'expliquer les motivations de l'inconscient collectif du peuple canadien-français. Trois études d'importance, publiées en un peu plus de deux ans, viennent raviver ce champ d'étude et, certainement, soulever quelques controverses.

Remaniement d'une thèse doctorale, Au commencement était le mythe de Victor-Laurent Tremblay en possède toute la profondeur intellectuelle mais également quelquefois les défauts inhérents aux exigences de ce travail universitaire (la constante justification des choix et méthodes étant sans doute la plus évidente). Son texte s'ouvre sur un heureux, utile mais très complexe survol de toutes les grandes théories reliées à l'étude du mythe (Durand, Girard, Bakhtine, Kristeva, Lévi-Strauss, Gans, Bateson, Propp, Bremond, Greimas, Bastide, etc.). Il apporte ainsi d'utiles éclaircissements sur les différences entre la mythanalyse, qui étudie les "manifestations du mythe à travers la culture afin d'en tirer non seulement le sens anthropologique, mais le sens sociologique et psychocritique".(p. 1), et la mythocritique, qui s'intéresse plutôt à toutes les représentations culturelles et sociales du mythe, et particulièrement à son application en littérature.

À partir des théories de Durand et Girard d'une part, et de Bakhtine d'autre part, Tremblay propose sa propre théorie psychosociale du mythe, laquelle sert de point de départ à une étude systématique de toutes les variantes traditionnelles du romanesque québécois: la légende folklorique, le roman d'aventures, le roman sentimental, le roman historique et, bien entendu, le roman du terroir. Chaque chapitre, qui se penche sur une ouvre en particulier, analyse tour à tour: (a) la forme romanesque de l'ouvrage étudié; (b) son contenu textuel et ; (c) sa contextualisation sociomythique. 
L'approche de Tremblay lui permet d'abord de découvrir, dans La Chasse-galerie (Honoré Beaugrand, 1900), un processus sacrificiel où s'affrontent deux types de victimisation qu'il nomme "intra " et "extra " communautaire, selon qu'ils s'adressent au transgresseur-adversaire (intra) ou à l'élément étranger au protagoniste principal (par la race, le sexe..., qualifié d'extra-communautaire). Le texte suivant, L'Influence d'un livre (Aubert de Gaspé fils, 1837), premier roman québécois, est analysé en fonction de l'union presque naturelle du roman d'aventures et de la tradition orale: Tremblay y voit un symbole de "mutation anthropologique nationale", où la collectivité se retrouve partagée entre le respect de l'ordre traditionnel et l'attrait pour le discours anticonformiste, axé principalement sur une critique des valeurs bourgeoises matérialistes.

Il poursuit son étude avec Les Anciens Canadiens (Aubert de Gaspé père, 1863), où il observe la présence d'un constant processus de réhabilitation du "mauvais double historique" (Arché). Dans le roman sentimental Angéline de Montbrun (Laure Conan, 1884), Tremblay relève, à travers l'emploi du procédé épistolaire et du journal intime, "le processus d'individualisation vers lequel tend de plus en plus "l'époque de Laure Conan (p. 279). Au commencement était le mythe se termine avec une brillante mythanalyse d'Un bomme et son péché (Claude-Henri Grignon, 1933) où il propose une vision originale de ce roman du terroir, avec comme hypothèse de référence la dialectique mythovictimaire dans laquelle la ville vient remplacer l'Anglais, longtemps "favorisé " par l'ultramontain, en tant que processus réunificateur.

Mythes et symboles dans la littérature québécoise ${ }^{2}$ d'Antoine Sirois débute à peu près, chronologiquement parlant, là où Tremblay s'était arrêté, et peut donc, au sens strict, être considéré comme un complément à l'ouvrage antérieur. Plutôt que d'approcher l'étude du mythe par les variantes du romanesque, Sirois préfère subdiviser son corpus selon la nature des mythes les plus constamment employés dans la littérature du Québec depuis le début du siècle et, conséquemment, depuis la vogue du roman du terroir. Son objectif n'est donc pas d'offrir une vue. d'ensemble de la littérature québécoise à partir d'une lecture du mythe mais bien d'apporter un éclairage particulier sur la façon dont l'écrivain contemporain perçoit et adapte certains mythes.

Cette approche implique une théorie de départ basée sur l'approche mythocritique (à l'opposé de l'approche mythanalytique favorisée par Tremblay), en ceci que l'auteur évacue tout l'espace sociologique et politique qui aurait pu engendrer la création et la production des 
textes. Cette décision consciente de l'auteur lui permet d'éviter du même coup de tomber dans la psychanalyse interprétative de l'inconscient des textes littéraires, mais entraîne toutefois la perte du contexte de création de l'écriture.

Son analyse la plus impressionnante demeure certainement celle de "Babylone contre Éden" (p. 25-42) où il scrute 61 romans du terroir créés par des auteurs canadiens-français (32 romans, 22 auteurs) et canadiens-anglais (29 romans, 20 auteurs). À l'instar de Tremblay, Sirois s'attarde sur la symbolique reliée à l'opposition ville/campagne ; l'intérêt de son étude réside toutefois dans son approche comparative qui pourrait certes obliger à une re-lecture idéologique des romans de la terre au Canada français. Toujours dans le domaine du terroir, Sirois procède également à une étude du mythe de la terre-mère, d'Homère à Zola et Ringuet, avant de s'attaquer à l'image de la cité maudite qu'est New York, à travers le roman Restons chez nous! de Damase Potvin.

Dans les chapitres suivants, l'auteur s'intéresse au mythe de la nature chez Gabrielle Roy, aux Parques et muses du Plateau MontRoyal si chères à Tremblay et à la Médée de Monique Bosco, avant d'entreprendre une descente aux enfers avec Héloïse d'Anne Hébert. Sirois rattache ensuite Le Ciel de Québec de Jacques Ferron avec SaintDenys Garneau, "un Orphée québécois " (p. 97). Finalement, les deux dernières études de l'auteur se concentrent sur la quête du père dans Le Cercle des arènes de Roger Fournier, et sur l'importance de la Genèse dans les romans d'Anne Hébert.

Moins attaché aux structures et délimitations reliées aux innombrables théories sur la mythocritique et la mythanalyse, l'ouvrage de Maurice Lemire ${ }^{3}$ s'inscrit dans une démarche de sensibilisation plus simple (mais non moins originale et efficace) à l'histoire littéraire du Québec. Il y fait preuve d'une immense érudition et d'une connaissance rarement égalée de la culture canadienne-française des $\mathrm{XvIII}^{\mathrm{e}}$ et $\mathrm{XIX}^{\mathrm{e}}$ siècles; influencé particulièrement par les théories de Northrop Frye sur le discours métaphorique (Anatomie de la critique) et par les hypothèses de Gilbert Durand sur l'interprétation de ces représentations (Anthropologie structurale de l'imaginaire), Lemire procède à une mythanalyse (au sens large) d'un imposant corpus littéraire canadienfrançais (qui recoupe souvent celui de V.-L. Tremblay) inscrit entre 1760 , date de la rupture avec la France, et 1867, qui coïncide avec le déclin intellectuel de la ville de Québec suite au déménagement des grandes institutions politiques du pays.

Son ouvrage se divise en cinq grands chapitres qui s'occupent chacun d'un grand thème représentant, selon lui, des "tentatives de 
réconciliation des contraires" (p. 17). La délimitation du cosmos québécois, d'un territoire propre, constitue son premier objet d'intérêt. Il y souligne la réactualisation constante des mythes primordiaux, l'anamnèsis, qui se manifeste d'abord, au Canada français, par l'allusion du peuple au célèbre "bon vieux temps", et à travers les contes, légendes et chansons si chères au peuple. Y est abordée l'inéluctable question de l'ici et de l'ailleurs où les notions de ville, de campagne et du territoire américain sont abondamment étudiées.

Une fois le territoire défini, Lemire cherche, dans un deuxième temps, à expliquer les liens qui se développent entre l'homme et sa patrie, laquelle présente une insoluble dichotomie entre le respect de la terre ancestrale et l'attachement aux grands espaces américains. Il y souligne la présence de deux grands mythes, ceux de la terre promise et de la quête, à son avis indissociables l'un de l'autre. S'y ajoute, chez les auteurs canadiens-français, la symbolique du voyage qui, contrairement à la tradition littéraire européenne, prend constamment une connotation négative.

Le quatrième chapitre (qui devrait logiquement être le troisième, comme l'indique l'introduction, erronée [p. 17]), se penche sur les liens qui doivent inévitablement se tisser entre les Canadiens et les premiers habitants de l'Amérique. L'auteur en profite pour dresser un historique du mythe de l'Indien américain, création littéraire des romantiques français du $x_{x}{ }^{e}$ siècle. Il scrute ensuite à la loupe la légende de l'Iroquoise, "le moule formel idéal dans lequel se coule le mythos indien canadien" (p. 155), avant d'aborder la question des manuels d'histoire du Canada et des légendes canadiennes.

Toujours dans le cadre des relations humaines, Lemire s'intéresse, dans un fascinant troisième chapitre, à l'interdit par excellence de la littérature canadienne-française des $\mathrm{XVIII}^{\mathrm{e}}$ et $\mathrm{XIX}^{\mathrm{e}}$ siècles : Éros et l'attrait de la chair, interdit qui ne peut être relevé, selon ses découvertes, que par la mort. À travers la littérature, il y observe avec minutie l'institution du mariage, d'amour ou d'affaire, avant de souligner et d'analyser la présence constante du groupe binaire amour/mort, qui va bien audelà de l'attrait romantique.

Légèrement isolé des autres, le cinquième et dernier chapitre de la Formation de l'imaginaire au Québec tente de réconcilier l'imaginaire savant, chasse gardée de l'élite des bourgeois et des lettrés, et l'envoûtant imaginaire populaire, où dominent la superstition, l'aventure et le fantastique. C'est donc par une étude de la littérarisation de ces contes et légendes populaires que Lemire démontre l'importance de ces récits dans la formation de l'identité collective. Il y souligne par ailleurs le 
rôle prépondérant joué à la fois par le narrateur intermédiaire et par les ultramontains dans ce passage de l'oral à l'écrit, alors que le conte populaire devint selon lui un "prolongement de la prédication" (p. 208-209). Voilà pourquoi il choisit de se concentrer, dans son analyse, sur le conte fantastique, forme préférée des libéraux, où abondent les pactes avec le diable, sous toutes ses formes, et les revenants, associés au thème de la fête, au défoulement et à la libération des tabous (p. 232).

Mythanalyse et mythocritique sont des outils fondamentaux à une meilleure appréciation de la littérature; les trois ouvrages recensés ici s'avèrent une richissime mine d'informations et apportent des éléments nouveaux et originaux dans l'interprétation des œuvres littéraires au Canada français et au Québec. Cette approche théorique véhicule toutefois avec elle le danger de sombrer dans une psychanalyse facile d'une collectivité et dans un respect trop grand pour les théoriciens et leur jargon souvent incompréhensible et inutile. Chacun de nos auteurs chute malheureusement, à l'occasion, dans l'un de ces deux pièges. Le lecteur doit donc apprendre à oublier (et pardonner!) l'emploi des eirônes, alazônes et bômolochoi (alors que les termes "timides", "imposteurs" et "bouffons", beaucoup plus clairs et parfaitement acceptables, faciliteraient tellement la lecture), les innombrables théories freudiennes qui cherchent à expliquer l'inconscient de personnages fictifs, et les applications à la lettre des structures schizomorphes et des dominantes posturales, copulatives et digestives qui, avouons-le, apportent peut-être beaucoup à la science de l'homme, mais bien peu à la compréhension de sa littérature. Il n'en demeure pas moins que plusieurs des études présentes dans ces ouvrages sont à la fois intelligentes, intéressantes et novatrices, et pourront certes servir de modèles aux analyses critiques à venir.

1. On peut en trouver une liste exhaustive dans l'ouvrage de Victor-Laurent Tremblay, Au commencement était le mvthe, Ottawa, Les Presses de l'Université d'Ottawa, 1991, p. 289-290,.

2. Antoine Sirois, Mythes et Symboles dans la littérature québécoise, Montréal, Triptyque, $1992,154 \mathrm{p}$.

3. Maurice Lemire, Formation de l'imaginaire littéraire au Québec 1764-1867, Montréal, l'Hexagone, 1993, 280 p. 